\title{
Caracterização das Pesquisas sobre Eficácia Coletiva Docente na Perspectiva da Teoria Social Cognitiva ${ }^{1}$
}

\author{
Maély Ferreira Holanda Ramos ${ }^{2}$ \\ Ana Patrícia Oliveira Fernandez \\ Fernando Augusto Ramos Pontes \\ Simone Souza Costa e Silva \\ Universidade Federal do Para
}

\begin{abstract}
RESUMO - A eficácia coletiva de professores refere-se ao modo como os membros do corpo docente julgam as capacidades desse grupo para realizar as tarefas específicas de sua função. Este estudo objetivou revisar sistematicamente pesquisas sobre a eficácia coletiva docente, na perspectiva social cognitiva. Para tanto, foram realizadas buscas de artigos publicados entre 2010 a 2014, na base de dados da CAPES. O levantamento resultou em 12 artigos que contemplavam os critérios de inclusão pré-estabelecidos. Os resultados indicaram, por meio da análise dos objetivos dos artigos selecionados, que as variáveis mais utilizadas para estudar a eficácia coletiva foram: autoeficácia e satisfação no trabalho. Notou-se ainda que a maioria das pesquisas utilizou abordagem quantitativa, sendo necessário ampliar os estudos, aplicando técnicas qualitativas.
\end{abstract}

Palavras-Chave: eficácia coletiva, professores, revisão sistemática

\section{Characterization of studies on collective teacher efficacy from a Social Cognitive Theory perspective}

\begin{abstract}
Collective efficacy of teachers refers to the way faculty members judge the capabilities of this group to perform the specific tasks of the ir function. This study aimed to systematic ally review studies on collective teacher efficacy from a social cognitive perspective. The refore, articles in the CAPES database published between 2010 to 2014 were reviewed .The survey resulted in 12 articles which contemplated pre-established inclusion criteria. The results indicated by means of analysis of the objectives of the articles that the variables most commonly used to study the collective teacher efficacy were: self-efficacy and job satisfaction. It was also noted that the majority of the studies useda quantitative approach, indicating a necessity to expand the studies applying qualitative techniques.
\end{abstract}

Keywords: collective efficacy, teachers, systematic review

De acordo com a Teoria Social Cognitiva de Bandura (1986), as pessoas, em parte, são produtos de seus ambientes, tendo, no entanto, capacidade para selecionar, criar e transformar as diversas circunstâncias ambientais às quais são expostas. Essa capacidade lhes permite influenciar cursos de acontecimentos, motivar, orientar e reorientar suas ações.

Bandura (1986) explica o funcionamento humano a partir de processos cognitivos, os quais são autorregulados e autorreflexivos, gerando adaptações e mudanças. Esses processos cognitivos são atividades cerebrais emergentes que exercem uma influência determinante no comportamento das pessoas. Isso porque a mente humana é geradora, criativa e reflexiva, não é apenas reativa. Nessa concepção, as pessoas não são dirigidas somente pelas circunstâncias ambientais ou por impulsos internos camuflados. Os indivíduos são agentes capazes de fazer coisas acontecerem de forma proativa, podendo exercer controle sobre as próprias vidas, estabelecendo limites para o autodirecionamento.

Na perspectiva social cognitiva, as crenças que o indivíduo desenvolve sobre si mesmo e, mais especificamente, sobre

1 Apoio: $\mathrm{CNPq}$

2 Endereço para correspondência: Barão do Triunfo, Ed. Uno Tower, apt. 301, Marco, Belém, Pará, CEP: 66095-050.E-mail: maelyramos@ hotmail.com sua capacidade de controlar seu próprio comportamento são fundamentais, pois interferem na tomada de decisão (Bandura, Azzi \& Polydoro, 2008). As crenças são fatores que funcionam como mecanismos de mudança de comportamento, influenciando-o por meio da expectativa de sucesso (Bandura, 1997).

No âmbito individual, a autoeficácia é a crença das pessoas sobre si mesmas, relacionada às tarefas desempenhadas, ao nível de esforço, à persistência, ao nível de estresse, etc. Para Bandura (1997), a autoeficácia refere-se aos julgamentos que as pessoas desenvolvem sobre suas próprias capacidades de organizar e executar ações. Essa crença oferece as bases para a motivação humana.

De acordo com a Teoria Social Cognitiva, as crenças de eficácia coletiva, por sua vez, referem-se ao modo como os membros de um grupo julgam as capacidades desse grupo para realizar determinadas atividades. Nessa perspectiva teórica, a eficácia coletiva é considerada como uma crença compartilhada que se desenvolve na percepção de cada membro sobre a capacidade dos outros indivíduos participantes do mesmo grupo (incluindo ele mesmo). Esse é um ponto chave na teoria, pois se considera a interatividade e a coordenação dinâmica de cada integrante para julgar as capacidades coletivas do grupo. Dessa forma, quanto 
menor for a variação entre os julgamentos dos indivíduos referente à eficácia do seu grupo, mais coesa será a crença, constituindo-se uma crença grupal compartilhada, que se torna uma estrutura normativa e cultural. Ressalta-se, no entanto, que, quando há intensa variação na percepção dos indivíduos referente à eficácia coletiva do grupo, isto pode indicar uma crença menos coesa, decorrente de problemas na dinâmica da interação dos membros, bem como na realização de tarefas (Bandura, 1986, 1997).

A dimensão de grupo, que caracteriza o construto de eficácia coletiva, procura explicar o comportamento a partir da capacidade de gerenciar ações e tarefas ao longo da vida. Assim, eficácia coletiva é definida como a crença partilhada de capacidades conjuntas para organizar e executar cursos de ações requeridos para se produzir determinados níveis de tarefas, bem como para superar falhas cometidas (Bandura, 1986, 1997).

A autoeficácia e a eficácia coletiva podem ser aplicadas a diferentes ambientes institucionais. Em relação à eficácia coletiva, seu aspecto organizacional diz respeito às capacidades de desempenho de um sistema social como um todo. Tal compreensão estende-se a empresas, grupos políticos, associações esportivas, instituições escolares e outros. Especificamente no campo escolar, tanto a autoeficácia quanto a eficácia coletiva devem ser analisadas e compreendidas, respeitando as características próprias do ambiente de ensino e das relações que se desenvolvem (Bandura, Azzi \& Polydoro, 2008).

\section{Crenças de Eficácia de Professores}

\section{Autoeficácia Docente}

As crenças de autoeficácia de professores são julgamentos que os professores, como indivíduos, desenvolvem sobre suas capacidades de alcançar resultados relacionados à aprendizagem e ao comportamento dos seus alunos. Essas crenças influenciam de forma importante as ações docentes (Gibson \& Dembo, 1984; Tschannen-Moran \& Hoy, 2001).

Esse construto tem despertado interesse em diversos pesquisadores (Ashton, Olejnik, Crocker, \&McAuliffe, 1982; Guskey, \& Passaro, 1994; Pajares, 2007; Tschannen-Moran, Woolfolk, \& Hoy, 1998), dado o poder preditivo acerca da prática docente e a influência sobre os resultados acadêmicos dos estudantes. As crenças de autoeficácia de professores têm sido relacionadas de forma positiva a vários fatores, como sucesso acadêmico, ensino mais eficaz, maior envolvimento da família (Gibson \& Dembo, 1984; Hoy \& Woolfolk, 1993), assim como motivação dos alunos (Bandura, 1997).

Professores que apresentam baixos níveis de senso de eficácia têm mais dificuldades para lidar com os obstáculos e desafios do cotidiano, além de maiores níveis de estresse relacionado ao trabalho (Betoret, 2006). Em contrapartida, professores com elevado senso de eficácia tendem a apresentar maiores níveis de satisfação no trabalho (Lee, Dedrick, \& Smith, 1991), capacidade para planejar melhor suas atividades, desenvolvendo estratégias de ensino mais adequadas (Allinder, 1994), assim como amostrarem-se mais autoconfiantes (Da Costa \& Riordan, 1996).

\section{Eficácia Coletiva de Professores}

Eficácia coletiva docente refere-se aos julgamentos dos professores de uma escola sobre a capacidade do conjunto de educadores de organizar e executar cursos de ação exigidos para se conseguir resultados positivos junto aos alunos, promovendo a aprendizagem e um melhor desempenho acadêmico (Goddard, Hoy, \& Hoy, 2004). Não por acaso, resultados de pesquisas indicam relação significativa entre eficácia coletiva de professores e resultados acadêmicos dos alunos (Bandura, 1993; Goddard, 2001, 2002; Klassen, Tze, Betts, \& Gordon, 2011; Mccoach \& Colbert, 2010).

Considerada como importante aspecto do contexto escolar (Bandura, 1993, 1997), a eficácia coletiva de professores pode explicar, em parte, os efeitos da ação docente sobre o sucesso acadêmico dos estudantes, de modo a contribuir para a compreensão de como as escolas apresentam resultados diferentes entre si (Goddard, Hoy \& Hoy, 2000). Vale destacar que não se pode perder de vista fatores que devem ser considerados nesse contexto, como os aspectos sociodemográficos (Goddard, 2001), socioeconômicos (Parker, 2006; Tchannen-Moran \& Barr, 2004), somados àqueles relacionados à infraestrutura, suporte pedagógico, questões sociais e políticas de cada escola (Adams \&Forsyth, 2006; Skaalvik \& Skaalvik, 2007).

O contexto escolar é um dos mais propícios para se compreender os efeitos das crenças de eficácia coletiva, uma vez que seus membros atuam de forma interdependente, visando a objetivos em comum. Entende-se que, em escolas, os professores atuam individualmente em suas tarefas específicas e coletivamente, visando às realizações institucionais (Bandura, 1993). Nesse espaço, são vivenciadas tanto dificuldades em comum, quanto sucessos alcançados, que influenciam as crenças acerca da capacidade de desempenhar ações em prol da instituição, também, atuando como produto da interação dinâmica dos membros do grupo.

Diferentes estudos constataram a influência exercida pelas crenças de eficácia coletiva de professores em vários aspectos do contexto escolar. Professores que acreditam na capacidade coletiva para ensinar seus alunos de forma eficaz, contribuindo para o desenvolvimento de sua escola (Goddard, Lo Gerfo, \& Hoy, 2004), sentem-se encorajados a partilhar decisões organizacionais (Ross, Hogaboam-Gray,\& Gray, 2003) e, consequentemente, exercem algum tipo de ação sobre as normas sociais do espaço escolar (Goddard, 2001). Níveis elevados de eficácia coletiva favorecem escolas com professores mais capazes de lidar com alunos que apresentam dificuldades de aprendizagem, de apoiar os pais e de tê-los como parceiros no processo ensino-aprendizagem (Bandura, 1997).

\section{Pesquisas sobre Eficácia Coletiva Docente}

As pesquisas sobre eficácia coletiva de professores pautam-se na compreensão desse constructo em suas relações 
com aspectos predominantes do contexto escolar, tais como o desempenho de alunos e a satisfação no trabalho. Essas pesquisas buscam entender a construção dessas crenças nas instituições de ensino.

Tschannen-Moran et al. (1998) realizaram uma revisão sistemática com a proposta de examinar os fundamentos conceituais e as ferramentas de mensuração referentes às crenças de eficácia de professores (autoeficácia e eficácia coletiva). Realizaram-se buscas em bases de dados relacionadas à área da psicologia, tais como PsycINFOeERIC, entre 1986 e 1997. Utilizou-se o termo teacher efficacy como único descritor, sendo que a maior parte dos achados foi caracterizada como artigos e livros. Encontraram-se 68 estudos investigando a autoeficácia docente em conjunto com outras variáveis, tais como: carreira docente (tempo de magistério), nível escolar (educação infantil, ensino fundamental, ensino médio) e contexto (ambientes rural, urbano e suburbano). O foco das buscas deteve-se em dois domínios teóricos específicos: fundamentos de eficácia de Rotter (1966) e de Bandura (1986). Nenhum artigo sobre eficácia coletiva docente foi identificado nessa revisão sistemática.

Sendo assim, a revisão de Tschannen-Moran et al. (1998) concentrou sua análise no constructo da autoeficácia, mas algumas importantes conclusões puderam ser feitas sobre as crenças de eficácia de grupo. Em relação à eficácia coletiva docente, os pesquisadores concluíram que essa temática estava sendo negligenciada e sugeriram que mais estudos fossem realizados, explorando as implicações nos resultados dos alunos. Percebeu-se a necessidade de ampliar as investigações sobre a relação da eficácia coletiva de professores com autoeficácia docente, bem como, também, com clima organizacional. Tschannen-Moran et al. (1998) evidenciaram, ainda, que essas crenças estão diretamente relacionadas à performance de grupo, sendo necessário, por isso, considerar o contexto social.

Klassen et al. (2011) realizaram revisão sistemática de artigos sobre eficácia coletiva e autoeficácia docente referentes ao período de 1998-2009. Os estudos foram selecionados a partir dos bancos de dados PsycINFO, Web of Ciência e ERIC. Ao final das buscas, 218 objetos foram analisados. Klassen et al. evidenciaram a pouca atenção que tem sido dada às fontes de eficácia coletiva de professores, sinalizando que é necessário ampliar os estudos nessa área. Perceberam que é preciso investigar como essas fontes podem variar no decorrer da carreira docente, avaliando as influências culturais (Cheung, 2008). Outro problema identificado foi a medição incorreta das crenças. Apesar das orientações dadas por Bandura (1997), ainda há problemas evidentes nas medidas utilizadas pelos pesquisadores, como a insuficiente base teórica dos instrumentos e o direcionamento incorreto das medidas, as quais devem refletir juízos de capacidade prospectiva e não juízos de intenção.

Desta forma, considerando o contexto apresentado, o presente trabalho se propõe a construir um panorama das pesquisas sobre eficácia coletiva de professores. Realizouse uma revisão sistemática de artigos publicados no período de 2010 a 2014.

\section{Método}

\section{Estratégias de Buscas e Análise}

Nesta revisão sistemática, realizou-se um levantamento de artigos, buscando caracterizar as pesquisas sobre eficácia coletiva de professores. Para tanto, prescindiu-se de sete etapas para a efetivação desta revisão: (a) formulação da pergunta/problema; (b) localização e seleção dos estudos; (c) avaliação crítica dos estudos; (d) coleta de dados nos artigos; (e) análise e apresentação dos dados; (f) interpretação dos dados; (g) aprimoramento e atualização da revisão.

$\mathrm{Na}$ primeira etapa, formulou-se a pergunta central por meio da técnica $\mathrm{PVO}$, em que $\mathrm{P}$ refere-se à situação problema, participantes ou contexto; $\mathrm{V}$ refere-se às variáveis do estudo; $\mathrm{O}$ aplica-se ao desfecho ou resultado esperado. Essa técnica permite organizar os elementos de uma pesquisa para melhor estruturar as perguntas. Dessa forma, com a técnica PVO, construiu-se a seguinte questão: Quais as principais características das pesquisas sobre satisfação no trabalho docente? Considera-se a seguinte estrutura: P (situação problema, participantes ou contexto) - professores em contexto escolar; V (variável do estudo) - eficácia coletiva; $\mathrm{O}$ (resultado esperado) - identificar as principais características dos estudos sobre a temática em questão.

A etapa 2 (localização e seleção dos estudos) envolveu a definição de alguns descritores relacionados à temática em questão. Nessa seleção, os autores basearam-se nos componentes da escala PVO e posteriormente realizaram consulta aos Descritores em Ciências da Saúde (DECS). Assim, instituíram-se os seguintes termos: collective efficacy, collective efficacy beliefs, teacher collective efficacy, collective perceptions, social cognitive theory, teacher efficacy.

Com a escolha dos descritores, construíram-se estratégias de busca para serem submetidas às bases de dados. Para tanto, aplicaram-se operadores booleanos aos componentes da escala PVO. Desta maneira, formaram-se duas estratégias de busca em português e inglês, em forma de equação, a saber:

- (collective efficacy OR collective efficacy beliefs OR collective perceptions OR efficacy beliefs) AND teacher AND school AND (theory social cognitive OR social cognitive theory).

- (eficácia coletiva $O R$ crenças de eficácia coletiva $O R$ percepções coletivas OR crenças de eficácia) AND (professor OR docente) $A N D$ escola $A N D$ teoria social cognitive.

Os artigos foram selecionados nas bases de dados disponíveis no portal da CAPES-Periódicos, que é uma das maiores bibliotecas virtuais do mundo. O portal CAPESPeriódicos resume conteúdo científico de alto nível, com publicações nacionais e internacionais, e contém artigos e revistas de diferentes áreas do conhecimento, entre outras produções.

Para avaliação dos artigos levantados ( $3^{\mathrm{a}}$ etapa), foram considerados os seguintes critérios de inclusão: conter no título o termo collective efficacy ou eficácia coletiva; estar completo e disponível; ter sido publicado nos últimos 5 anos (2010 a 2014); ser redigido em inglês ou português; ter sido 
revisado por pares; e ter como foco investigar o constructo da eficácia coletiva de professores (artigos teóricos e empíricos) a partir da Teoria Social Cognitiva, ou seja, estudos que consideraram a eficácia coletiva como crenças compartilhas e desenvolvidas a nível de indivíduo. Optou-se por selecionar apenas artigos com os termos de busca nos títulos para que fossem incluídos somente os estudos que tratassem de forma central do construto investigado. Foram excluídos todos os achados que não obedeceram aos critérios de inclusão mencionados. Consideraram-se apenas artigos dos últimos cinco anos, pois a última revisão sistemática sobre eficácia coletiva docente, na perspectiva social cognitiva, abrangeu o recorte temporal de 1998 a 2009 (Klassen et al., 2011). As buscas resultaram em apenas 12 artigos que contemplaram todos os critérios de inclusão pré-determinados.

Procedeu-se à coleta de dados nos artigos a partir de suas principais características ( $4^{\mathrm{a}}$ Etapa). Ressalta-se que, neste estudo, não foram analisados os resultados dos artigos selecionados, pois o foco estava na caracterização geral das pesquisas sobre eficácia coletiva docente, explorando elementos de identificação (palavras-chave) e aspectos metodológicos. A organização dos dados estruturou-se em cinco categorias de análise: (a) análise das palavraschave - para investigar possíveis conexões entre os artigos a partir dos seus descritores; (b) objetivos dos artigos para identificar as metas de investigação e as principais variáveis dos estudos; (c) natureza e formas de abordagem do problema de pesquisa - para identificar a frequência de estudos empíricos, teóricos, qualitativos, quantitativos e mistos, bem como o nível de análise dos artigos (individual ou coletivo); (d) procedimentos técnicos e classificação do método - para identificar a forma como os dados dos artigos foram levantados e para classificar os métodos em longitudinais ou transversais; e (e) instrumentos de coleta referentes aos instrumentos que foram mais utilizados nos estudos para medir a eficácia coletiva docente: questionários, roteiros e escalas.

Os dados foram extraídos, utilizando-se roteiro contendo as informações detalhadas de cada estudo. Assim sendo, para levantamento e análise dos dados ( $5^{\mathrm{a}}$ Etapa), foram utilizados os seguintes programas: (a) EXCEL 2010 e SPSS 20 - para levantamento e realização de uma estatística de tendência central que informa como a amostra é em sua totalidade ou em média (Dancey \& Reidy, 2006); (b) MENDELEY - para organização das referências; (c) NodeXL - para construção de grafos, criando representações geométricas das conexões entre as palavras-chave dos artigos; e (d) NVIVO 10 - para desenvolvimento de análise de conteúdo aplicada aos objetivos dos artigos selecionados para esta revisão sistemática.

Um grafo é composto por vértices (pontos/elemento) e arestas (ligações entre os pontos/conexões), para representar associações entre elementos de qualquer natureza (Matos, 2013; Pinheiro, 2013). Nesta revisão, fez-se uso dessa técnica para investigar as conexões entre as palavras-chave. Os grafos possuem medidas (métricas) específicas que auxiliam na investigação das conexões entre os elementos em estudos.

Para a análise com grafos, foram calculadas as métricas: (a) Grau de Centralidade (degree) - para quantificar o número de ligações associadas a um elemento; e (b) Centralidade
Autovetor(Eigenvector Centrality) - para medir o grau de importância de um vértice (Matos, 2013; Pinheiro, 2013).

Após a organização e análise dos dados, foi realizado o processo de interpretação das informações, a partir da Teoria Social Cognitiva. Posteriormente deu-se a construção e o aprimoramento da revisão sistemática ( $6^{\mathrm{a}}$ e $7^{\mathrm{a}}$ etapas).

\section{Resultados e Discussão}

\section{Análise das Palavras-Chave}

No processo de caracterização dos artigos, realizou-se a análise das conexões entre as palavras-chave dos estudos levantados nesta revisão sistemática por meio de grafos. Assim, utilizou-se a ferramenta NodeXL, que é indicada para calcular métricas capazes de identificar as interações dinâmicas entre os elementos que compõem o conjunto de variáveis de interesse do pesquisador, bem como gerar representações geométricas, em forma de diagramas, resultantes das relações estabelecidas (Smith, 2013). Dessa forma, a Figura 1 apresenta o grafo das palavras-chave e suas conexões.

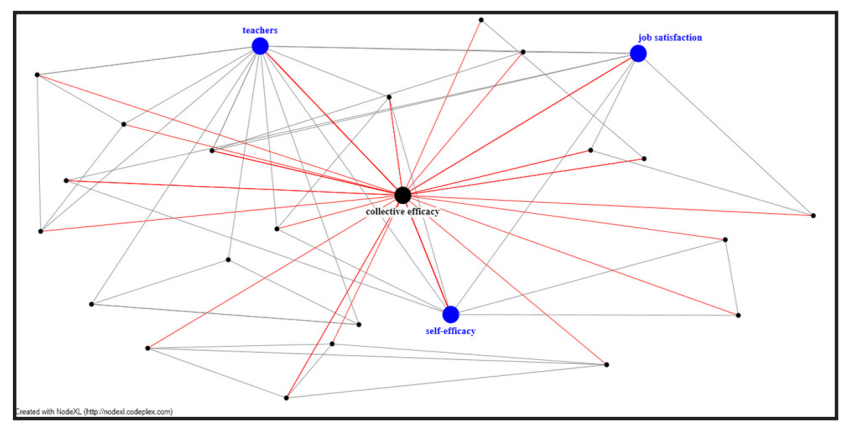

Figura 1. Grafo das palavras-chave e suas conexões

A Figura 1 é um grafo que ilustra a conexão entre as 25 palavras-chave identificadas nesta revisão sistemática. Ressalta-se que os pontos (vértices) representam os descritores e as linhas (arestas) indicam as conexões que se estabeleceram. Foram destacadas, por discos maiores, as palavras-chave mais importantes conectadas à eficácia coletiva, considerando-se como ponto de corte termos com métrica de Centralidade Autovetor $>0,06$. Essa métrica calcula os elementos mais relevantes a partir da capacidade de conexão com outros vértices. Assim, elencaram-se os seguintes descritores com seus respectivos graus de importância: collective efficacy $(0,1)$; teacher $(0,08)$; selfefficacy $(0,06)$; job satisfaction $(0,06)$.

Por ser o principal termo de busca desta revisão sistemática, a palavra-chave collective efficacy alcançou maior escore de importância, assim como demonstrou maior capacidade de conexão com outros termos (pontos) do grafo, estabelecendo ocorrência conjunta com outros 21 descritores. Essa capacidade de conexão foi calculada pela métrica Grau de Centralidade. Essa métrica quantifica o número de conexões que se estabelecem entre os pares de vértices (descritores). Notou-se que, por ser o principal termo de busca, a palavra-chave collective efficacy foi identificada em 
91\% dos artigos. Ressalta-se, no entanto, que o referido termo não alcançou $100 \%$ das pesquisas, pois o critério de inclusão pré-estabelecido indica que o termo de busca deveria constar no título do artigo, visando selecionar apenas estudos que investigassem a eficácia coletiva docente de forma central, o que se confirmou após a análise dos artigos.

$\mathrm{O}$ termo teacher foi o que alcançou a maior frequência de ocorrência conjunta (6) com o vértice collective efficacy, pois foram selecionados apenas artigos que tratassem da eficácia coletiva aplicada aos professores. O segundo termo com maior ocorrência conjunta com collective efficacy foi a palavra-chave self-efficacy (5). Isto sugere que a relação entre autoeficácia e eficácia coletiva docente foi um dos principais focos de análise nos artigos investigados. O vértice job satisfaction, também em destaque no grafo, totalizou apenas três ocorrências conjuntas com collective efficacy. As demais palavras-chave totalizaram apenas uma ocorrência conjunta cada. De forma geral, os resultados da análise das palavras-chave sugerem conexões entre os artigos a partir das suas palavras-chave.

\section{Objetivos dos Estudos}

Para categorização e estudo dos objetivos dos artigos investigados, aplicou-se análise de conteúdo por meio do software NVIVO 10. Essa ferramenta foi desenvolvida para facilitar técnicas qualitativas, visando promover uma melhor organização, análise e visualização dos dados. Com o mapeamento dos objetivos, pretendeu-se identificar quais os principais interesses teóricos e empíricos das pesquisas sobre eficácia coletiva docente nos últimos cinco anos (2010 a 2014), a partir da base de dados selecionada para esta revisão sistemática. Para tanto, os objetivos dos artigos em inglês foram traduzidos e inseridos no NVIVO 10.

Procedeu-se à codificação dos dados, que consiste em reunir informações por tópicos, temas ou casos, criando "nós" (categorias). Assim, classificaram-se todas as fontes de informações (objetivos) inseridas no software (Figura 2).

A Figura 2 consiste em um modelo exploratório gerado pelo NVIVO 10 a partir da codificação dos objetivos dos artigos, apresentando a estrutura das categorias encontradas. Indicou-se o conjunto de variáveis utilizadas pelos pesquisadores para investigar a eficácia coletiva docente. Os modelos exploratórios gerados pelo NVIVO 10 evidenciam

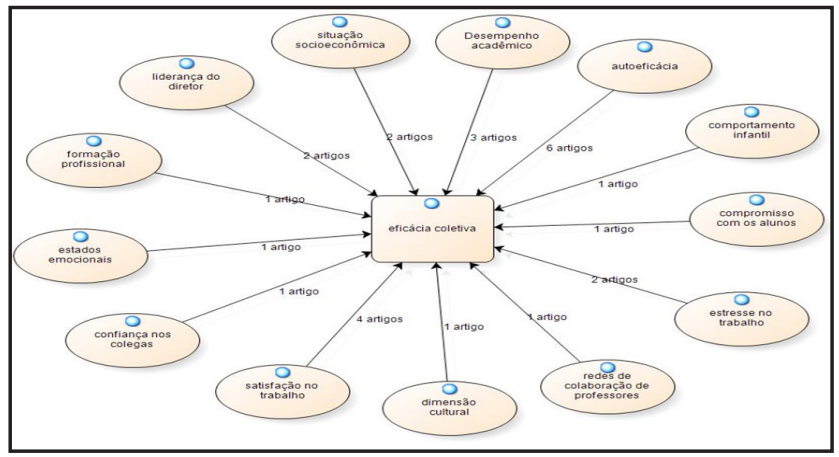

Figura 2. Modelo exploratório: estrutura de categorias encontradas os padrões que se estabelecem por meio da categorização realizada pelo pesquisador, construindo mapas conceituais.

A análise dos objetivos dos estudos que fazem parte desta revisão sistemática revela que a eficácia coletiva docente foi investigada em conjunto com as seguintes variáveis: (a) desempenho acadêmico - refere-se ao aproveitamento escolar dos alunos em sistemas institucionais de avaliação (Lee, Zhang, \& Yin, 2011); (b) autoeficácia - é a crença do professor na sua própria capacidade para exercer com eficácia a sua função (Viel-Ruma, Houchins, Jolivette, \& Benson, 2010); (c) comportamento infantil - refere-se ao comportamento disciplinar dos alunos nas séries iniciais do Ensino Fundamental (Lee et al., 2011);(d) estresse no trabalho - aplica-se ao estresse decorrente da rotina escolar no cumprimento da docência (Klassen, 2010); (e) redes de colaboração de professores - são as redes sociais de apoio que se estabelecem no ambiente de trabalho (Moolenaar, Sleegers, \& Daly, 2012);(f) dimensão cultural - referente as características culturais de coletivismo ou individualismo (Klassen, Foster, Rajani, \& Bowman, 2009); (g) satisfação no trabalho - é o grau em que as pessoas gostam da função que realizam (Duffy \& Lent, 2009); (h) confiança nos colegas - é a confiança que um indivíduo tem no seu grupo (Lee et al., 2011); (i) estados emocionais - refere-se às experiências emocionais dos professores no ambiente escolar que podem influenciar o comportamento futuro (Stephanou, 2013); (j) formação profissional - aplica-se a uma contínua capacitação dos professores, visando ao aprimoramento da prática docente (Lee et al., 2011); (k) liderança do diretor - refere-se às atitudes de liderança de diretores de escolas, visando contribuir para a melhoria do desempenho docente e discente (Kurt, Duyar, \& Çalik, 2012)

A Figura 2 indica, ainda, o número de artigos que investigou as diferentes variáveis em conjunto com a eficácia coletiva docente. Destaca-se que as categorias não são exclusivas de um único estudo, uma vez que um mesmo artigo pode ter investigado uma ou mais variáveis. Sendo assim, a soma da frequência das categorias ultrapassa o número total de artigos levantados nessa revisão sistemática (12). Notouse que a autoeficácia foi a variável mais investigada em conjunto com a eficácia coletiva docente ( 6 artigos), seguida por: satisfação no trabalho (4), desempenho acadêmico (3), liderança do diretor (2), estresse no trabalho (2) e situação socioeconômica (2). As demais categorias foram exploradas em apenas um artigo cada.

Com o NVIVO 10, foi possível verificar a frequência das palavras por meio da ferramenta "consulta", que oferece ao pesquisador diferentes opções para visualizar os dados, buscando identificar padrões e categorias. Assim, gerou-se uma representação gráfica da frequência dos termos em forma de nuvem de palavras para facilitar a percepção das principais categorias contidas nos objetivos dos artigos (Figura 3).

A nuvem de palavras evidencia o grau de importância de um termo, considerando sua frequência na base de dados. Logo, quanto maior for a palavra, mais frequente ela é nas fontes de informações. Ressalta-se que essa técnica considera os elementos em forma de palavras simples. Notou-se, considerando apenas os termos referentes às categorias de análise, que a autoeficácia e a satisfação no trabalho alcançaram maior destaque. Isto se coaduna com os dados das 


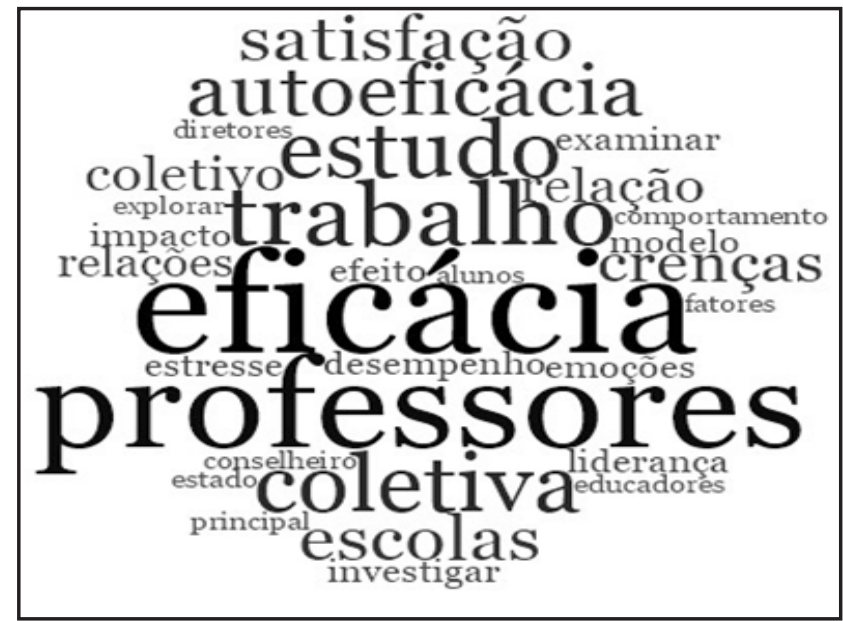

Figura 3. Nuvem de palavras dos objetivos dos artigos

frequências das categorias que sugerem um maior interesse dos pesquisadores por analisar a relação dessas variáveis com a eficácia coletiva docente.

A literatura tem indicado que a autoeficácia e a eficácia coletiva docente são construtos distintos, porém interdependentes. Bandura (1997) utilizou o termo causalidade recíproca para indicar a relação de mão dupla ao interpretar a associação entre as variáveis.

Estudos sugerem que eficácia coletiva e a satisfação no trabalho são variáveis que também podem se influenciar mutuamente. Stephanou (2013) investigou a relação entre essas variáveis numa amostra de 268 professores do Ensino Fundamental de 85 escolas estaduais de várias regiões da Grécia. Os resultados indicaram que a autoeficácia e a satisfação no trabalho estabelecem uma causalidade recíproca, influenciando-se mutuamente.

\section{Natureza da Pesquisa e Forma de Abordagem do Problema}

Os dados levantados revelaram que, quanto à natureza das pesquisas, 11 estudos foram realizados em caráter empírico, seguidos de um artigo de revisão sistemática. A concentração das pesquisas em estudos empíricos se divide em 10 que adotaram a abordagem quantitativa e um com abordagens qualitativas. Esses resultados apontam para um domínio das abordagens quantitativas nessa base de dados. Não foram identificados estudos com abordagem mista (quanti-quali).

Portanto, apenas um estudo utilizou, exclusivamente, pesquisa qualitativa. Putneye Broughton (2011) investigaram a relação entre eficácia coletiva de professores e desempenho de alunos, utilizando a visão de Vygotsky (1978, 1986, 1997) sobre o desenvolvimento individual e coletivo. Nesse artigo, os pesquisadores aplicaram entrevista, uma técnica qualitativa, para captar as crenças coletivas e individuais dos professores e seus impactos na comunidade escolar.

Pesquisadores da área têm indicado a necessidade de intensificar os estudos com abordagens qualitativas, advertindo para a importância de se buscarem alternativas de investigação para além das abordagens quantitativas. Estudos com técnicas qualitativas, como o estudo de caso, por exemplo, permitiriam aprofundar, ainda mais, a compreensão de como essas crenças operam (Tschannen-Moran et al., 1998; Henson, 2002; Klassen et al., 2011).

Ainda referente a esse tópico, buscou-se avaliar o nível de análise estabelecido pelos pesquisadores para investigar a eficácia coletiva docente, identificando se a abordagem teórica e empírica se deu em nível individual ou grupal. Notou-se que $100 \%$ dos artigos levantados nesta revisão sistemática analisaram o referido construto com enfoque teórico e empírico no nível grupal, na medida em que a percepção dos professores é compartilhada. Deve-se ressaltar, no entanto, que não há uma "mente coletiva” e que a percepção se desenvolve no indivíduo (Bandura, 1997). Esse dado indica que todas as pesquisas revisadas se adequaram aos pressupostos teóricos da Teoria Social Cognitiva no estudo da eficácia coletiva docente.

\section{Procedimentos Técnicos e Classificação dos Métodos}

Em continuidade à caracterização dos artigos levantados nesta revisão sistemática, foram analisados os procedimentos técnicos e os métodos dos estudos. Para a categorização dos métodos, considerou-se a classificação de Cozby (2003), a saber: métodos transversal e longitudinal. No método transversal, os participantes das pesquisas são estudados num ponto do tempo apenas. No método longitudinal, observa-se o mesmo grupo de indivíduos em diferentes épocas. Assim, identificou-se que 100\% dos estudos empíricos levantados nesta revisão sistemática adotaram métodos transversais.

Frequentemente, o método transversal é mais utilizado que o longitudinal, pois é menos dispendioso em termos financeiros e os resultados úteis são alcançados de forma mais rápida. Tais características têm atendido às necessidades de muitos pesquisadores (Cozby, 2003). Contudo, assim como os estudos qualitativos, as pesquisas longitudinais podem ajudar a conduzir as investigações sobre a eficácia do professor a um nível mais elevado de maturação, porém, têm sido negligenciadas (Klassen et al., 2011).

$\mathrm{Na}$ análise dos procedimentos técnicos utilizados nos artigos sobre eficácia coletiva do professor, identificaramse as seguintes categorias: (a) pesquisa de levantamento com escalas; (b) entrevista. A pesquisa de levantamento com escalas destacou-se com uma acentuada frequência de utilização, sendo a técnica escolhida por todos os pesquisadores nos estudos com abordagens quantitativas. A entrevista foi a técnica utilizada pelo único artigo que adotou uma abordagem qualitativa.

A partir dos dados supracitados, é possível perceber o panorama das técnicas utilizadas nas pesquisas analisadas, deixando nítida a diferença existente na frequência do uso de cada técnica, com prevalência significante de estudos quantitativos e da utilização de pesquisa de levantamento com escalas.

\section{Instrumento de Coleta de Dados}

Na identificação dos instrumentos de coleta de dados para medir a eficácia coletiva docente, notou-se que a CE-Scale 
(longform), de Goddard et al. (2000), foi a mais utilizada, sendo aplicada em quatro estudos (Kurt et al., 2012; McCoach \& Colbert, 2010; Viel-Ruma et al., 2010;). Essa escala, tipo likert, mede as crenças de eficácia coletiva de professores através de 21 itens, em um contínuo de 1 a 6 . Goddard et al. (2000) utilizaram a Teacher Efficacy Scale de Gibson e Dembo's (1984) como base para desenvolver as medidas da CE-Scale (longform) (Dantas, Guerreiro-Casanova, \& Azzi, 2012).

Os itens dessa escala foram construídos focando as crenças coletivas, ao invés das crenças individuais de eficácia de professores, utilizando uma perspectiva de orientação compartilhada de grupo para melhor refletir as experiências coletivas no desenvolvimento da escala. Goddard et al. (2000) criaram itens positivos e negativos para não influenciar os participantes da pesquisa. Esses itens estão relacionados à "competência de grupo" (GC) e à "análise de tarefas" (AT) (Dantas et al., 2012).

O segundo instrumento mais utilizado foi a Collective Teacher Efficacy Belief Scale (CTEBS), de Tschannen-Moran e Barr (2004), aplicado em 3 estudos(Klassen, Usher, \& Bong, 2010; Klassen, 2010; Lee et al., 2011). Essa escala possui 12 itens, num contínuo de 1 a 9, e visa mensurar as crenças de eficácia coletiva a partir das percepções grupais sobre as práticas de ensino e a disciplina dos alunos (Dantas et al., 2012).

O terceiro instrumento mais utilizado nos artigos foi a CE-Scale (short form), de Goddard (2002), aplicada em 2 estudos (Gibson, Dembo,1984; Moolenaar et al., 2012). Esse instrumento é a forma mais curta da CE-Scale (longform) e foi criada com a finalidade de aprimorar a primeira escala, sendo constituída por 12 itens, num contínuo de 1 a 6 . O objetivo foi construir itens mais parcimoniosos, calibrando as questões quanto a GC e AT. O melhoramento dessa medida foi realizado, pois a primeira escala, em versão longa, demostrou possuir uma distribuição desigual dos itens (não parcimoniosa), com prevalência das questões relacionadas à GC (13) sobre as questões voltadas à investigação da AT (8) (Dantas et al., 2012).

Apenas um estudo utilizou o grupo de subescalas criado por Caprara, Barbaranelli, Borgogni \& Steca (2003) para medir a eficácia coletiva, bem como outras variáveis: autoeficácia percebida e satisfação no trabalho. A subescala respectiva à eficácia coletiva foi composta por 9 itens, num contínuo de 1 a 7 (Stephanou, 2013).

\section{Conclusões e Sugestões para Futuras Pesquisas}

O mapeamento dos dados coletados na presente revisão sistemática permitiu identificar as principais tendências concernentes aos aspectos metodológicos dos artigos selecionados para o estudo das crenças de eficácia coletiva de professores. Além disso, possibilitou a investigação das conexões estabelecidas entre as palavras-chave dos artigos.

A aplicação de análise com grafo e métricas de centralidade permitiu identificar os descritores com maior frequência de ocorrência conjunta com a eficácia coletiva docente: teacher, self-efficacy, job satisfaction. A conexão dessas palavras-chave com o termo de busca collective efficacy, em um número maior de artigos, sugere que os artigos investigados nesta revisão sistemática investiram, teórica e empiricamente, na investigação da eficácia coletiva em conjunto com a autoeficácia e a satisfação no trabalho.

Isso foi confirmado na análise dos objetivos dos artigos. No tópico em questão, observou-se que as variáveis autoeficácia e satisfação no trabalho foram consideradas em investigação conjunta com a eficácia coletiva na maioria dos artigos, respectivamente $50 \%$ e $33 \%$. Outras variáveis também foram consideradas: desempenho de alunos, situação socioeconômica, comportamento infantil, estresse no trabalho, redes de colaboração, dimensão cultural, satisfação no trabalho, confiança nos colegas, estados emocionais, formação profissional e liderança de diretores.

Os aspectos metodológicos aqui investigados indicaram a necessidade de ampliação das pesquisas com abordagens qualitativas e mistas, entendendo que podem favorecer a melhor compreensão da eficácia coletiva docente. Não foram encontrados estudos longitudinais, entendendo-se que pesquisas dessa natureza podem oferecer maiores informações quanto à formação e ao desenvolvimento das crenças de eficácia coletiva em longo prazo, assim como investigar a variação das mesmas no início, decorrer e término da carreira docente.

Notou-se ainda, no estudo dos instrumentos utilizados pelos pesquisadores para avaliar as crenças de eficácia coletiva, que houve prevalência de pesquisas de levantamento com escalas (84\%), sendo que a CE-scale (longform) de Goddard et al. (2000) foi a mais utilizada (4 artigos). Destacase que, nos 12 estudos levantados nesta revisão sistemática, foram identificadas apenas quatro diferentes escalas de avaliação da eficácia coletiva docente, sendo estas: $C E$-scale (longform) de Goddard et al. (2000); CE-scale (shortform) de Goddard (2002); CTEBS, de Tschannen-Moran e Barr (2004); grupo de subescalas criado por Caprara, Barbaranelli, Borgogni \& Steca (2003).

Algumas limitações nos resultados apresentados nesta revisão sistemática deram-se em função dos critérios de inclusão, já que as buscas dos artigos científicos se deram apenas na base de dados da CAPES e excluíram-se outras fontes de informações, tais como teses e dissertações. Futuros pesquisadores podem explorar teses e dissertações, entre outras fontes de informações não investigadas aqui, além de avaliar outras bases de dados.

Outra limitação deste estudo diz respeito ao recorte teórico escolhido. A literatura sobre eficácia coletiva é vasta, por esse motivo não se exploraram artigos que analisaram o conceito potência, que se refere ao fenômeno coletivo voltado para as crenças grupais de equipes. Futuras pesquisas podem abranger essas questões.

Considera-se que é necessário o fortalecimento e a ampliação das pesquisas sobre as crenças coletivas, buscando despertar o interesse por investigações que expliquem melhor como essas percepções se desenvolvem. Os resultados alcançados apontam não só para os avanços, mas também para as lacunas e problemas que ainda são emergentes nesse campo de investigação e, nesse sentido, esses achados oferecem orientações para futuras pesquisas. 


\section{Referências}

Adams, C. M., \& Forsyth, P. B. (2006). Proximate sources of collective teacher efficacy. Journal of Education Administration, 44, 625-642. doi:10.1108/09578230610704828

Allinder, R. (1994). The relationship between efficacy and the instructional practices of special education teachers and consultants. Teacher Education and Special Education, 17, 86-95. doi: 10.1177/088840649401700203

Ashton, P. T., Olejnik, S., Crocker, L., \& McAuliffe, M. (1982). Measurement problems in the study of teachers'sense of efficacy. In Annual Meeting of the American Educational Research Association. New York. doi: 10.3102/00346543068002202

Bandura, A. (1986). Social foundations of thought and action - A Social Cognitive Theory. Englewood Cliffs, N.J.: Prentice Hall.

Bandura, A. (1993). Perceived self-efficacy in cognitive development and functioning. Educational Psychologist, 28, 117-148.

Bandura, A. (1997). Self-efficacy: The exercise of control. New York: W. H. Freeman.

Bandura, A., Azzi, R. G., \& Polydoro, S. (2008). Teoria Social Cognitiva: Conceitos básicos. Porto Alegre, RS: Artmed.

Betoret, F. D. (2006). Stressors, self-efficacy, coping resources, and burnout among secondary school teachers in Spain. Educational Psychology, 26, 519-539. doi: 10.1080/01443410500342492

Caprara, G. V., Barbaranelli, C., Borgogni, L., \& Steca, P. (2003). Efficacy beliefs as determinants of teachers' job satisfaction. Journal of Educational Psychology, 95(4), 821-832. doi:10.1037/0022-0663.95.4.821

Cheung, H. Y. (2008). Teacher efficacy: A comparative study of Hong Kong and Shanghai primary in- service teachers. Australian Educational Researcher, 35, 103-123. doi:10.1007/ BF03216877

Cozby, P. C. (2003). Métodos de pesquisa em ciência do comportamento. São Paulo: Atlas.

Da Costa, J. L., \& Riordan, G. (1996). Teacher efficacy and the capacity to trust. In Annual Meeting of the American Educational Research Association. New York.

Dancey, C. P., \& Reidy, J. (2006). Estatística sem matemática para psicologia. Porto Alegre, RS: Artmed.

Dantas, M. P, Guerreiro-Casanova, D. C., \& Azzi, G. R. (2012). Eficácia Coletiva de professores: Análise de escalas internacionais de avaliação. AvaliaçãoPsicológica, 11, 181190.

Duffy, R. D., \& Lent, R. W. (2009). Test of a social cognitive model of work satisfaction in teachers. Journal of Vocational Behavior, 75(2), 212-223. doi:10.1016/j.jvb.2009.06.001

Gibson, S., \& Dembo, M. H. (1984). Teacher efficacy: A construct validation. Journal of Educational Psychology, 76(4), 569-582. doi:10.1037//0022-0663.76.4.569

Goddard, R. D. (2001). Collective efficacy: A neglected construct in the study of schools and student achievement. Journal of Educational Psychology, 93, 467-476. doi:10.1037//00220663.93.3.467

Goddard, R. (2002). A theoretical and empirical analysis of the measurement of collective efficacy: The development of a short form. Educational and Psychological Measurement, 62(1), 97-110. doi:10.1177/0013164402062001007
Goddard, R. D., Hoy, W. K., \& Hoy, A. W. (2000). Collective teacher efficacy: Its meaning, measure, and impact on student achievement. American Educational Research Journal, 37(2), 479-507. doi:10.3102/00028312037002479

Goddard, R. D., Hoy, W. K., \& Hoy, A. W. (2004). Collective efficacy beliefs: Theoretical developments, empirical evidence, and future directions. Educational Researcher, 33, 3-13. doi:10.3102/0013189X033003003

Goddard, R. D., LoGerfo, L., \& Hoy, W. K. (2004). High school accountability: The role of perceived collective efficacy. Educational Policy, 18, 403-425. doi:10.1177/0895904804265066

Guskey, T. R., \& Passaro, P.D. (1994). Teacher Efficacy: A study of construct dimensions. American Educational Research Journal, 31, 627-643. doi: 10.3102/00028312031003627

Henson, R. K. (2002). From adolescent angst to adulthood: Substantive implications and measurement dilemmas in the development of teacher efficacy research. Educational Psychologist, 37, 137-150.doi: 10.1207/S15326985EP3703

Hoy, W. K., \& Woolfolk, A. E. (1993). Teachers' sense of efficacy and the organizational health of schools. Elementary School Journal, 93, 335-372.

Klassen, R. M., Foster, R. Y., Rajani, S., \& Bowman, C. (2009). Teaching in the Yukon: Exploring teachers' efficacy beliefs, stress, and job satisfaction in a remote setting. International Journal of Educational Research, 48(6), 381394. doi:10.1016/j.ijer.2010.04.002

Klassen, R. M. (2010). Teacher stress: The mediating role of collective efficacy beliefs. The Journal of Educational Research, 103(5), 342-350. doi:10.1080/00220670903383069

Klassen, R. M., Usher, E. L., \& Bong, M. (2010). Teachers' collective efficacy, job satisfaction, and job stress in crosscultural context. The Journal of Experimental Education, 78(4), 464-486. doi:10.1080/00220970903292975

Klassen, R. M., Tze, V. M. C., Betts, S. M., \& Gordon, K. A. (2011). Teacher efficacy research 1998-2009: Signs of progress or unfulfilled promise? Educational Psychology Review, 23(1), 21-43. doi:10.1007/s10648-010-9141-8

Kurt, T., Duyar, I., \& Çalik, T. (2012). Are we legitimate yet?: A closer look at the casual relationship mechanisms among principal leadership, teacher self-efficacy and collective efficacy. Journal of Management Development, 31(1), 71-86. doi:10.1108/02621711211191014

Lee, V. E., Dedrick, R., \& Smith, J. (1991). The effect of the social organization of schools on teachers' efficacy and satisfaction. Sociology of Education, 64, 190-208.

Lee, J. C., Zhang, Z., \& Yin, H. (2011). A multilevel analysis of the impact of a professional learning community, faculty trust in colleagues and collective efficacy on teacher commitment to students. Teaching and Teacher Education, 27(5), 820-830. doi:10.1016/j.tate.2011.01.006

Matos, I. M. D. de. (2013). Teoria dos grafos no ensino básico e secundário. (Universidade de Aveiro). Recuperado de https:// owl.english.purdue.edu/owl/resource/560/09/

McCoach, D. B., \& Colbert, R. D. (2010). Factors underlying the collective teacher efficacy scale and their medianting role in the effect of socioeconomic status on academic achievement at the school level. Measurement and Evoluation in Couseling and Development, 43, 31-47. 
Moolenaar, N. M., Sleegers, P. J. C., \& Daly, A. J. (2012). Teaming up: Linking collaboration networks, collective efficacy, and student achievement. Teaching and Teacher Education, 28(2), 251-262. doi:10.1016/j.tate.2011.10.001

Pajares, F. (2007). Culturalizing educational psychology. In F. Salili \& R. Hoosain (Eds.), Culture, motivation, and learning (pp. 75-100). Charlotte: Information Age.

Parker, K. (2006). Collective teacher efficacy, pupil attainment and socio-economic status in primary school(R). Improving Schools, 9(2), 111-129. doi:10.1177/1365480206064965

Pinheiro, J. M. V. (2013). A investigação e as redes de conhecimento na European Network for Housing Research. Recuperado de http://repositorio-aberto.up.pt/handle/10216/72175

Putney, L. G., \& Broughton, S. H. (2011). Developing collective classroom efficacy: The teacher's role as community organizer. Journal of Teacher Education, 62(1), 93-105. doi:10.1177/0022487110381760

Ross, J. A., Hogaboam-Gray, A., \& Gray, P. (2003). The contribution of prior student achievement and school processes to collective teacher efficacy in elementary schools. In Annual Meeting of the American Educational Research Association. Chicago.

Rotter, J. B. (1966). Generalized expectancies for internal versus external control of reinforcement. PychologicalMonogyztphs, $80,1-28$.

Smith, M. A. (2013). NodeXL: Simple network analysis for social media. In International Conference on Collaboration Technologies and Systems (CTS).San Diego, CA, USA.
Skaalvik, E.M., \& Skaalvik, S. (2007). Dimensions of teacher selfefficacy and relations with strains factors, perceived collective teacher efficacy and teacher burnout. Journal of Educational Psychology, 99, 611-625. doi:10.1037/0022-0663.99.3.611

Stephanou, G. (2013). The role of teachers' self- and collectiveefficacy beliefs on their job satisfaction and experienced emotions in school. Psychology, 4(3), 268-278. doi:10.4236/ psych.2013.43A040

Tschannen-Moran, M., \& Barr, M. (2004). Fostering student learning: The relationship of collective efficacy and student achievement. Leadership and Policy in Schools, 3, 189-209.

Tschannen-Moran, M., \& Hoy, A. W. (2001). Teacher efficacy: Capturing an elusive construct. Teaching and Teacher Education, 17(7), 783-805.

Tschannen-Moran, M., Woolfolk, H. A., \& Hoy, W. K. (1998). Teacher efficacy: Its meaning and measure. Review of Educational Research, 68, 202-248.

Viel-Ruma, K., Houchins, D., Jolivette, K., \& Benson, G. (2010). Efficacy beliefs of special educators: The relationships among collective efficacy, teacher self-efficacy, and job satisfaction. Teacher Education and Special Education: The Journal of the Teacher Education Division of the Council for Exceptional Children, 33(3), 225-233. doi:10.1177/0888406409360129

Vygotsky, L. S. (1978). Mind in society: The development of higher psychological processes. Cambridge, MA: Harvard University Press.

Vygotsky, L. S. (1986). Thought and language. Cambridge, MA: MIT Press.

Vygotsky, L. S. (1997). Educational psychology. Boca Raton, FL: St. Lucie Press.
Recebido em 13.11.2013

Primeira decisão editorial em 31.10.2014

Versão final em 09.12.2014

Aceito em 08.07.2015 